\title{
Polyphenylene-Based Solid Acid as an Efficient Catalyst for Activation and Hydration of Alkynes
}

\author{
Yiyun Liu, Bolun Wang, Liqun Kang, Apostolos Stamatopoulos, Hao Gu, and Feng Ryan Wang*
}

Cite This: Chem. Mater. 2020, 32, 4375-4382

Read Online

ABSTRACT: Porous polymer catalysts possess the potential to combine the advantages of heterogeneous and homogeneous catalysis, namely, easy postreaction recycling and high dispersion of active sites. Here, we designed a $-\mathrm{SO}_{3} \mathrm{H}$ functionalized polyphenylene (PPhen) framework with purely $\mathrm{sp}^{2}$-hybridized carbons, which exhibited high activity in the hydration of alkynes including challenging aliphatic substrates such as 1-octyne. The superiority of the structure lies in its covalent crosslink in the $x y$ plane with a $\pi-\pi$ stacking interaction between the planes, enabling simultaneously high swellability and porosity $\left(653 \mathrm{~m}^{2} \cdot \mathrm{g}^{-1}\right)$. High acidic site density $\left(2.12 \mathrm{mmol} \cdot \mathrm{g}^{-1}\right)$ was achieved under a mild
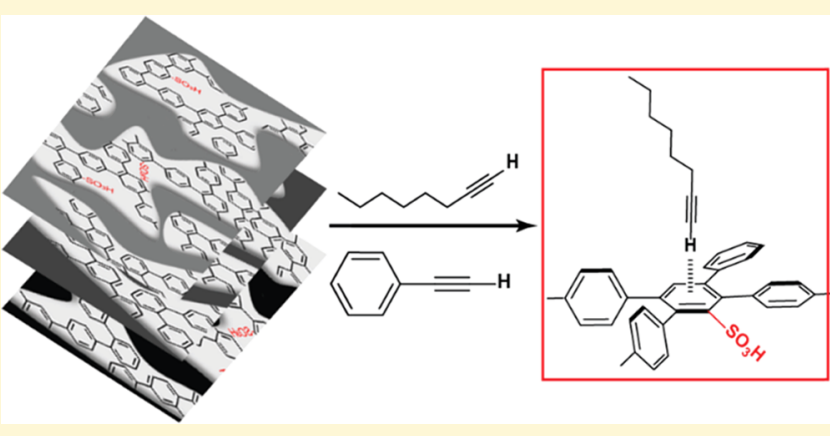
sulfonation condition. Similar turnover frequencies $(0.015 \pm 0.001$ $\min ^{-1}$ ) were obtained regardless of acidic density and crosslink content, suggesting high accessibility for all active sites over PPhen. In addition, the substituted benzene groups can activate alkynes through a T-shape $\mathrm{CH} / \pi$ interaction, as indicated by the 8 and 16 $\mathrm{cm}^{-1}$ red shift of the alkyne $\mathrm{C}-\mathrm{H}$ stretching peak for phenylacetylene and 1-octyne, respectively, in the infrared (IR) spectra. These advantages render $\mathrm{PPhen}-\mathrm{SO}_{3} \mathrm{H}$ a promising candidate as a solid catalyst replacing the highly toxic liquid phase acids such as the mercury salt.

\section{INTRODUCTION}

Porous organic materials play an important role in gas storage and separation, ${ }^{1,2}$ drug release, ${ }^{3}$ catalysis, sensing, ${ }^{4}$ and membrane separation ${ }^{5}$ due to their properties in merging the benefits of porosity, processability, synthesis diversity, and organic nature. ${ }^{6}$ A number of novel polymeric frameworks have been developed in recent years, ${ }^{6,7}$ such as polytriazine, ${ }^{8-10}$ hyper-cross-link polystyrene (HPS), ${ }^{11,12}$ nanoporous polydivinylbenzene (PDVB), ${ }^{13}$ dihydroxybenzoic acid-based polymer nanospheres, ${ }^{14}$ and porphyrin network polymers. ${ }^{15,16}$ The polymeric structures not only offer a high surface area and well-controlled porosity but also provide an organic environment, the advantage of lightweight, and a dynamic structure upon external stimulation. Thus, they have offered an innovative way to combine the advantages of homogeneous and heterogeneous catalysis. ${ }^{17}$ On one hand, a solvent-like reaction environment is established via organic ligands and the geometric structure of the framework, providing well-dispersed high-density active sites. On the other hand, the heterogeneous nature of solid catalysts facilitates postreaction separation, rendering them environment-friendly alternatives for highly toxic homogeneous liquid catalysts.

Various polymer-based solid acid catalysts have been investigated in the recent years for hydrolysis, ${ }^{18}$ hydration, ${ }^{19-21}$ dehydration, ${ }^{22-24}$ and other acid-catalyzed reactions ${ }^{25,26}$ in both gaseous and liquid phase as the replacement of liquid acids, which leads to severe technical and environ- mental problems in practical applications. Acid-catalyzed alkyne hydration is a straightforward and atom-economical method for the synthesis of ketones via carbocation intermediates, ${ }^{27}$ where efforts have been paid to replace the traditional highly toxic mercury salt catalysts with recoverable and environment-friendly solid acid catalysts. ${ }^{28,29}$ A number of attempts have been made in developing polymer-based solid acids; however, their catalytic activity is often limited by the contradiction lying within the nature of the polymeric framework.

A good polymeric heterogeneous catalyst requires high active site density and good accessibility simultaneously, which can be achieved in porous polymers by increasing the porosity and swellability. While the porosity of a polymer generally increases as a function of crosslink content, as seen in $\mathrm{sp}^{3}$ carbon linker groups, ${ }^{12}$ a three-dimensionally crosslinked framework reduces its ability to swell, and thus decreases the accessibility of the active sites. High swellability is usually obtained in polymers with lower crosslink content, ${ }^{30,31}$ within

Received: April 25, 2020

Revised: April 30, 2020

Published: May 1, 2020 
Scheme 1. Concept of PPhen Structure Showing the Covalent Crosslinks in $x y$-Plane (Left), Swelling Along $z$ Axis (Middle), and Activation of $\mathrm{C} \equiv \mathrm{C}-\mathrm{H}$ (Right) via T-Shape $\mathrm{CH} / \boldsymbol{\pi}$ Interaction $^{1}$
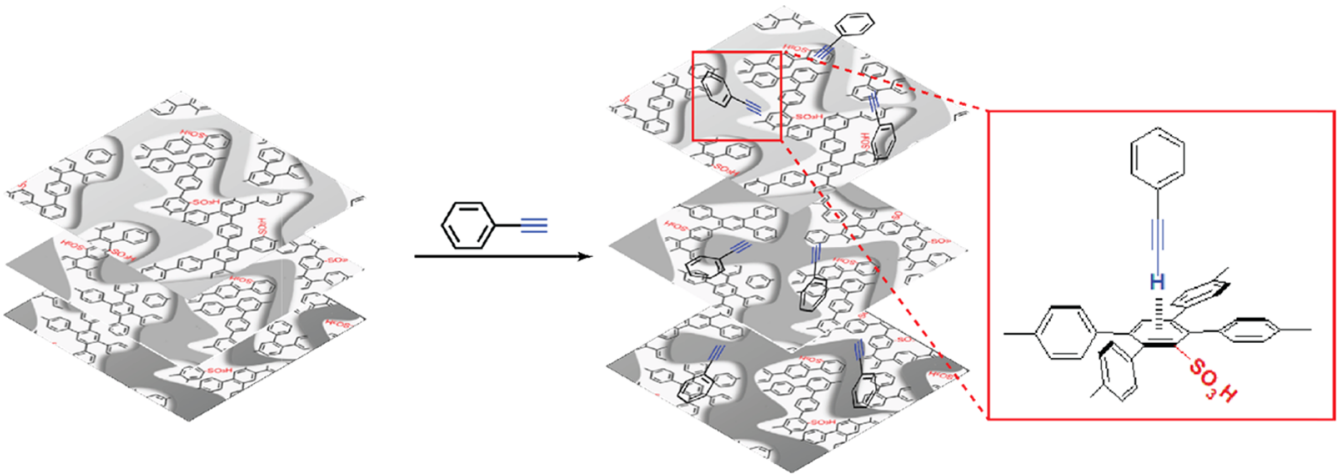

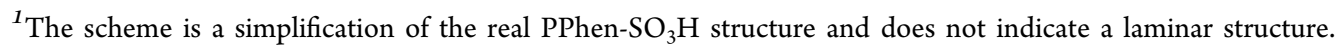

which pore formation is difficult due to close stacking of the linear chains. This further hinders the formation of highdensity active sites in the polymer matrix. Such a dilemma between high active site density and good molecular accessibility limits the practical application of porous polymers. Here, we hypothesize that the solution to this dilemma lies in the design of a polymer structure with covalent crosslinks at a two-dimensional level. This structure enables pore formation with rigid chemical bonds and pure molecular interactions between crosslinking layers, which provides good swellability, allowing reactant molecules to diffuse into the polymer matrix, which lowers the mass transfer limitation. ${ }^{25}$

To achieve this target, we employed the polyphenylene (PPhen)-based polymeric framework functionalized with $-\mathrm{SO}_{3} \mathrm{H}$ acidic sites. The material has been investigated previously as the ion-exchange resin, which shows that it can facilitate water diffusion through the hydrophilic regions and possesses a rigid backbone preventing the folding of the structure. $^{32,33}$ All carbon atoms in the PPhen-based polymer are $\mathrm{sp}^{2}$-hybridized, retaining covalent crosslinks in the $x y$-plane (Scheme 1 , left), while $\pi-\pi$ stacking becomes the main force at $z$ axis (Scheme 1, middle). As a result, PPhen achieves a high surface area of $653 \mathrm{~m}^{2} \cdot \mathrm{g}^{-1}$ and good swellability. Meanwhile, it also provides an organic solvent-like environment for reaction molecules via high density of disubstituted (phenylene) and tetrasubstituted benzene groups, ${ }^{34}$ which activate alkynes through the edge-to-face configuration (Scheme 1, right). ${ }^{35}$ The catalytic behavior of sulfonated PPhen solid acid is evaluated by the hydration of phenylacetylene and 1-octyne, two model compounds to study the structure-reactivity relationship and the activations of aromatic and aliphatic alkynes, respectively. A maximum of $2.12 \mathrm{mmol} \cdot \mathrm{g}^{-1}$ acidic site density was achieved under a mild sulfonation condition with sulfuric acid treatment at $40{ }^{\circ} \mathrm{C}$. The conversion of phenylacetylene reached $80 \pm 4 \%$ with $87 \pm$ $3 \%$ selectivity toward acetophenone at $100{ }^{\circ} \mathrm{C}$, which is three times higher than that of the commercial solid acid catalyst Amberlyst 15. A linear increase in the hydration reactivity with increasing $-\mathrm{SO}_{3} \mathrm{H}$ site density over PPhen- $\mathrm{SO}_{3} \mathrm{H}$ is achieved, resulting in similar turnover frequencies (TOF) regardless of acidic density and crosslink content. This result suggests good accessibility of all $-\mathrm{SO}_{3} \mathrm{H}$ sites for phenylacetylene. In addition, the electrophilic phenylene and tetrasubstituted benzene groups in the PPhen framework weaken the $\mathrm{C} \equiv \mathrm{C}$ bond, showing an $8 \mathrm{~cm}^{-1}$ red shift in the alkyne $\mathrm{C}-\mathrm{H}$ stretching for phenylacetylene and $16 \mathrm{~cm}^{-1}$ for 1-octyne. As a result, $\mathrm{PPhen}-\mathrm{SO}_{3} \mathrm{H}$ shows superior hydration activity even for aliphatic 1-octyne, a reaction that is very challenging for solid acid catalysts. ${ }^{21,36}$ The PPhen- $\mathrm{SO}_{3} \mathrm{H}$ catalyst has the potential to replace $\mathrm{Hg}$-based catalysts in hydration reactions.

\section{MATERIALS AND METHODS}

Synthesis of PPhen. To obtain a fully crosslinked PPhen-100\% polymer framework, 1,2,4,5-tetrabromobenzene $(1.531 \mathrm{~g}, 3.89 \mathrm{mmol})$ and benzene-1,4-diboronic acid $(1.289 \mathrm{~g}, 7.78 \mathrm{mmol})$ were added into $120 \mathrm{~mL}$ of dimethylformamide. The mixture was degassed through three freeze-pump-thaw cycles. $\mathrm{K}_{2} \mathrm{CO}_{3}(2.0 \mathrm{M}, 15 \mathrm{~mL})$ and $\mathrm{Pd}\left(\mathrm{PPh}_{3}\right)_{4}(0.3 \mathrm{~g}, 0.25 \mathrm{mmol})$ were then added with three subsequent freeze-pump-thaw cycles. The mixture was then purged with $\mathrm{Ar}$ and heated to $150{ }^{\circ} \mathrm{C}$ for $20 \mathrm{~h}$ under stirring. The product precipitated in water and was washed by water, dichloromethane, and methanol. Approximately $600 \mathrm{mg}$ of a gray product was obtained in each batch. PPhen with different crosslink contents were obtained by changing the ratio of 1,2,4,5-tetrabromobenzene and 1,4-dibromobenzene. The reactants used in the synthesis of different polymers are summarized in Table S1.

Synthesis of PPhen- $\mathrm{SO}_{3} \mathrm{H}$ Catalysts. As-prepared PPhen polymers were treated with $\mathrm{HCl} / \mathrm{H}_{2} \mathrm{O}_{2}(5: 1, \mathrm{v} / \mathrm{v})$ solution to remove $\mathrm{Pd}$ residue from the cross-coupling reaction. The mixture was stirred overnight under room temperature and heated up to $60{ }^{\circ} \mathrm{C}$ for $2 \mathrm{~h}$. The product was then washed with water and methanol. After drying in a vacuum oven overnight, $200 \mathrm{mg}$ of $\mathrm{H}_{2} \mathrm{O}_{2}$-treated polymer was added to $5 \mathrm{~mL}$ of concentrated sulfuric acid (98 wt \%) and stirred at $40{ }^{\circ} \mathrm{C}$ for $16 \mathrm{~h}$. The product was poured into $200 \mathrm{~mL}$ of deionized water and filtered, followed by washing with $\mathrm{H}_{2} \mathrm{O}$ and methanol until neutral $\mathrm{pH}$ to eliminate physically absorbed sulfuric acid molecules within the pores. The obtained $\mathrm{PPhen}-\mathrm{SO}_{3} \mathrm{H}$ catalysts were dried in a vacuum oven under $50{ }^{\circ} \mathrm{C}$ overnight.

Hydration Reaction of Alkynes. In a typical reaction, $20 \mathrm{mg}$ of catalyst was added into a glass tube reactor with $1 \mathrm{~mL}$ of $\mathrm{H}_{2} \mathrm{O}$ and $110 \mu \mathrm{L}(1 \mathrm{mmol})$ of phenylacetylene. Forty microliters $(0.176 \mathrm{mmol})$ of dodecane was also added into the reactor as the standard in the gas chromatography-mass spectrometry (GC-MS) measurement. The standard reaction continued for $24 \mathrm{~h}$ at $100{ }^{\circ} \mathrm{C}$. After the reaction, 5 $\mathrm{mL}$ of tetrahydrofuran (THF) was added and the mixture was placed in the ultrasonic washer for $30 \mathrm{~min}$. Fifty microliters was taken from the mixture and diluted with THF to be measured with GC-MS for quantification. The conversion of phenylacetylene, yield of acetophenone, and selectivity of catalysts mentioned are summarized in Table S4. Hydration reactions with the same molar amount of 1octyne were carried out under the same conditions. In the catalyst recycling experiments, the catalysts were separated by filtration, washed with ethanol, dried, and weighed. 


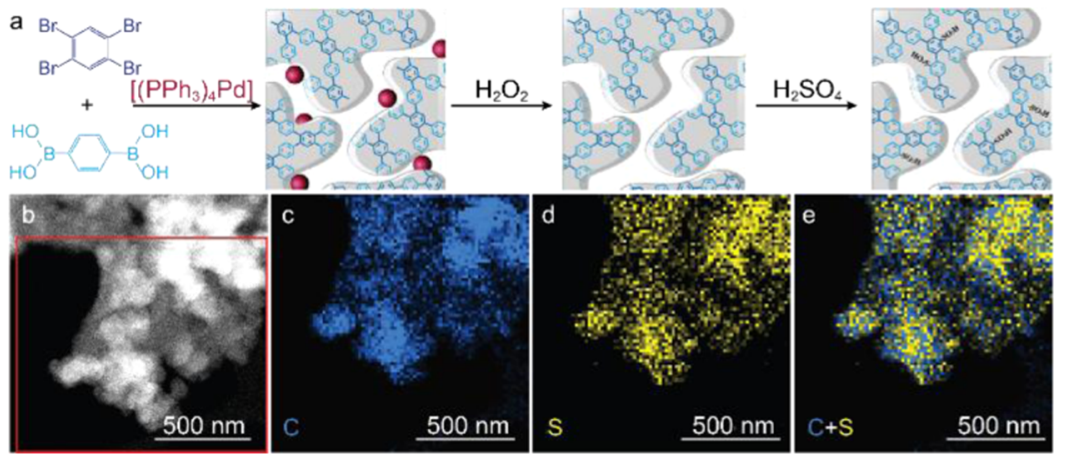

Figure 1. (a) Schematic diagram shows the synthesis of PPhen and loading of $\mathrm{SO}_{3} \mathrm{H}$. (b) HAADF-STEM image of PPhen-SO $\mathrm{H}$. (c-e), EDS map of carbon (c), sulfur (d), and carbon + sulfur (e). Region difference between STEM and EDS map is due to sample drifting.
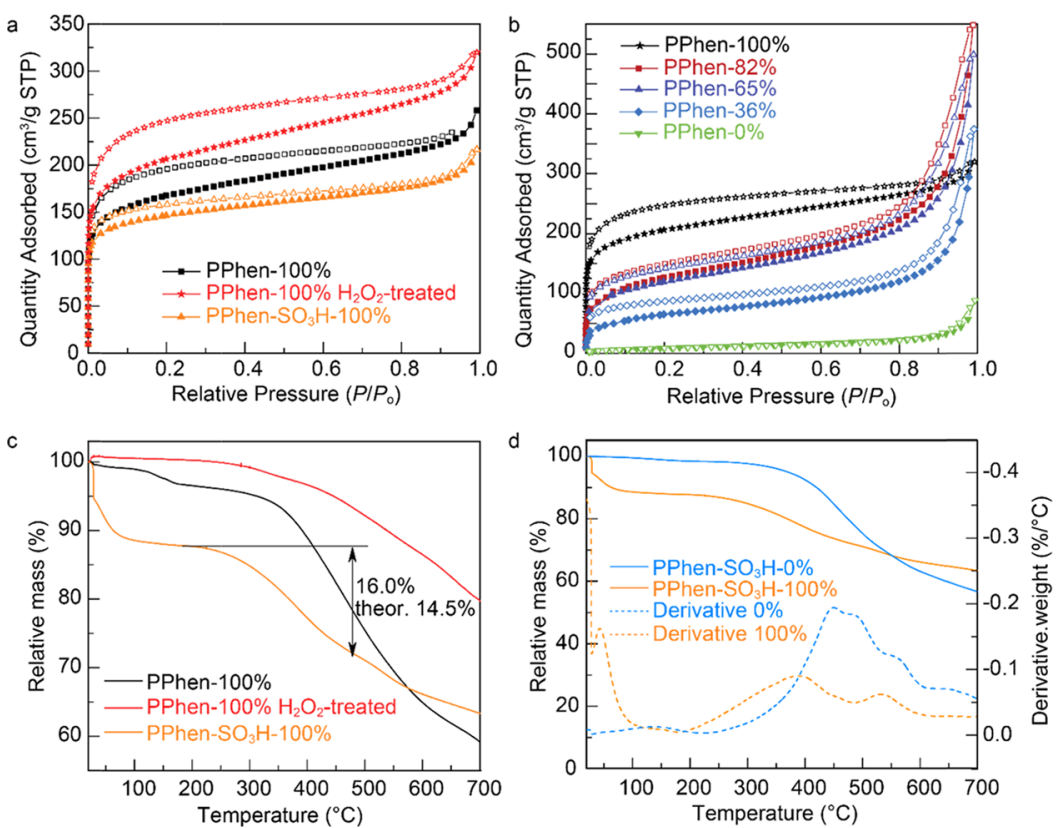

Figure 2. (a) $\mathrm{N}_{2}$ physisorption of fresh PPhen-100\% (black), PPhen-100\% treated with $\mathrm{H}_{2} \mathrm{O}_{2}$ (red), and PPhen-SO $\mathrm{H}-100 \%$ (orange). (b) Comparison of $\mathrm{N}_{2}$ physisorption for $\mathrm{H}_{2} \mathrm{O}_{2}$ treated PPhen-100, PPhen-82, PPhen-65, PPhen-36, and PPhen-0\%. (c) Thermogravimetric analysis (TGA) of the corresponding PPhen polymers in (a). (d) TGA and the first derivatives of weight loss for PPhen- $\mathrm{SO}_{3} \mathrm{H}-100 \%$ and $\mathrm{PPhen}^{-} \mathrm{SO}_{3} \mathrm{H}-0 \%$.

Characterizations. Samples for high-angle annular dark-field scanning transmission electron microscopy (HAADF-STEM) were prepared by sprinkling a small amount of dry sample powder on 400 mesh copper grids with a lacey carbon support film. High-resolution aberration-corrected HAADF-STEM images in Figures 1 and S2 and S4 were acquired on probe-corrected (CEOS) JEM ARM 200CF (JEOL, Japan) operated at $200 \mathrm{keV}$. We used a $40 \mu \mathrm{m}$ probe-forming aperture, resulting in $31.8 \mathrm{mrad}$ probe convergence semiangle. The HAADF signal was gathered at $8.0 \mathrm{~cm}$ STEM camera length, integrating the scattered electron intensity between 71.5 and 257.8 mrad. Energy-dispersive X-ray spectroscopy (EDS) data were obtained on the probe-corrected JEM ARM 200CF (JEOL, Japan), which is equipped with large solid-angle dual EDS detectors for X-ray spectroscopy and elemental mapping. The EDS data acquisition was carried out in STEM imaging mode, with a probe current of $143 \mathrm{pA}$ (probe size is 5C), with the microscope operated at $200 \mathrm{keV}$ acceleration voltage. Each EDS spectrum image is $100 \times 100$ pixels in size with $0.05 \mathrm{~s}$ exposure time per pixel. Gatan Microscopy Suite Software was used for EDS spectrum imaging data acquisition. Nitrogen adsorption-desorption isotherms were recorded at $77 \mathrm{~K}$ using a Micromeritics 3Flex surface characterization analyzer. The samples were degassed in vacuum at $200{ }^{\circ} \mathrm{C}$ overnight for removal of any contaminates. Specific surface areas were determined according to the BET model. Commercial Amberlyst 15 (20-30 mesh) is used as the benchmark for the hydration reactions, as it has been widely used as the solid acid catalyst for the transformation of organic molecules. ${ }^{37-40}$ Acidic site densities of as-synthesized PPhen-SO3H catalysts and Amberlyst 15 are measured via chemical titration. Fifty milligrams of the catalyst is added to $50 \mathrm{~mL}$ of $0.01 \mathrm{~mol} \cdot \mathrm{L}^{-1} \mathrm{NaHCO}_{3}$ solution and stirred for $24 \mathrm{~h}$. The mixture was filtered to get a clear solution, which is then titrated with $0.01 \mathrm{~mol} \cdot \mathrm{L}^{-1} \mathrm{HCl}$ solution to get the amount of remaining $\mathrm{NaHCO}_{3}$. The concentration of the $\mathrm{HCl}$ standard solution is determined by the titration with dry $\mathrm{Na}_{2} \mathrm{CO}_{3}$ standard. The concentration of $\mathrm{NaHCO}_{3}$ is then titrated with $\mathrm{HCl}$ standard solution. $\mathrm{NaHCO}_{3}$ is chosen because the scale of cross section for $\mathrm{HCO}_{3}{ }^{-}$ions $(3.0 \AA)$ is comparable to the reactants phenylacetylene $(4.3 \AA)$ and 1-octyne $(1.7 \AA)$ (estimated from the bond length in Lange's Handbook of Chemistry). Further, considering the nonpolar interaction between the reactants and organic substrate of polymer, the acidic sites included in the titration should be accessible to the reactants.

Thermogravimetric analysis (TGA) was measured on PerkinElmer Pyris 1 TGA. $2-10 \mathrm{mg}$ of the sample is heated from 30 to $700{ }^{\circ} \mathrm{C}$ with a heating rate of $10{ }^{\circ} \mathrm{C} \cdot \mathrm{min}^{-11}$. Attenuated total reflection infrared (ATR-IR) spectroscopy is measured with Bruker $\alpha$ FTIR spectrometer. The spectra were taken within the $4000-400 \mathrm{~cm}^{-1}$ range. The spectra of polymers absorbed with phenylacetylene were taken with $10 \mathrm{mg}$ of polymers absorbed with $5 \mu \mathrm{L}$ of phenylacetylene. 


\section{RESULTS AND DISCUSSION}

PPhen- $\mathrm{SO}_{3} \mathrm{H}$ Catalysts: Structure and Chemical Properties. PPhen is synthesized through the palladiumcatalyzed cross-coupling reaction of 1,2,4,5-tetrabromobenzene and benzene-1,4-diboronic acid as reported previously. ${ }^{34}$ To study the role of crosslink content in catalysis, 1,2,4,5tetrabromobenzene is selectively replaced with 1,4-dibromobenzene. This results in a controlled ratio between phenylene and tetrasubstituted benzene groups in the PPhen framework and thus determines the crosslink content, which is denoted as PPhen- $\mathrm{SO}_{3} \mathrm{H}-\mathrm{X} \%$, where $\mathrm{X} \%$ is the normalized crosslink content (Table S1, Supporting Information). To avoid the influence of Lewis acidity of $\mathrm{Pd}$ in the hydration reactions, asprepared PPhen is treated with $\mathrm{H}_{2} \mathrm{O}_{2}-\mathrm{HCl}$ to remove the Pd residue (Figure 1a). $-\mathrm{SO}_{3} \mathrm{H}$ sites are introduced by treating PPhen with concentrated sulfuric acid (95-97\%) (Figure 1a), the duration of which decides the acidic site density over the fully crosslinked polymer (PPhen- $\mathrm{SO}_{3} \mathrm{H}-100 \%$ ). The crosslinked polymer has irregular shapes in the form of curved aggregates (Figure S1, Supporting Information). High-angle annular dark-field-scanning transmission electron microscopy (HAADF-STEM) images show the candy floss shape of the polymer particles in the range of $100-400 \mathrm{~nm}$ (Figures $1 \mathrm{~b}$ and $\mathrm{S} 2$, Supporting Information). $-\mathrm{SO}_{3} \mathrm{H}$ sites disperse uniformly across the PPhen framework, as confirmed by energydispersive X-ray spectroscopy (EDS) element mapping of C and $\mathrm{S}$ (Figures $1 \mathrm{c}-\mathrm{e}$ and $\mathrm{S} 3$, Supporting Information). The linear polymer (PPhen- $\mathrm{SO}_{3} \mathrm{H}-0 \%$ ) has very similar morphology and $-\mathrm{SO}_{3} \mathrm{H}$ distributions (Figure $\mathrm{S} 4$, Supporting Information). PPhen- $\mathrm{SO}_{3} \mathrm{H}-100 \%$ shows an amorphous structure, as indicated in the X-ray diffraction (XRD) measurement (Figure S5, Supporting Information). The absence of a Pd signal in the EDS spectrum and the XRD pattern confirms the complete removal of Pd residues.

The $\mathrm{N}_{2}$ adsorption-desorption isotherm of fresh fully crosslinked PPhen (PPhen-100\%) (Figure 2a, black) shows a type I shape of isotherm, corresponding with microporous materials. The increase in adsorbed volume between 0.2 and $0.8 \mathrm{P} / \mathrm{P}_{0}$ indicates a large external surface, contributing to roughly $10 \%$ of the BET-equivalent specific surface area. ${ }^{34}$ Though BET surface area calculations are not representative for microporous materials, here, they are used to make a relative comparison between different PPhen systems. The steep increase of $P / P_{0}=0.9$ stems from the interparticulate void. The removal of the Pd residues by $\mathrm{H}_{2} \mathrm{O}_{2}$ not only reduces the weight of PPhen but also creates new pores and voids, which boosts the specific surface area from 529 to $653 \mathrm{~m}^{2} \cdot \mathrm{g}^{-1}$ (Figure 2a and Table S2, Supporting Information). Noticeably, the adsorption and desorption branches do not close completely. This phenomenon is consistent with other polymer isotherms in the literature $\mathrm{r}^{34,41-44}$ and has been attributed to the roughness within the mesopores. ${ }^{26}$ Sulfonation reduces the specific surface area to $461 \mathrm{~m}^{2} \cdot \mathrm{g}^{-1}$ with closed adsorption and desorption branches and small hysteresis (Figure 2a, orange). A decrease of crosslink content reduces specific surface areas to $435,407,224$, and $36 \mathrm{~m}^{2} \cdot \mathrm{g}^{-1}$ for PPhen-82, PPhen-65, PPhen-36, and PPhen-0\%, respectively (Figure $2 \mathrm{~b}$ and Table S1, Supporting information). In contrast to PPhen-100\%, the surface area of PPhen- $0 \%$ increases from 36 to $76 \mathrm{~m}^{2} \cdot \mathrm{g}^{-1}$ after sulfonation (Figure S6 and Table S2, Supporting Information). This is possibly due to the increased repulsive force between $-\mathrm{SO}_{3} \mathrm{H}$ sites in linear chains, creating new pore systems.

The PPhen polymer framework shows outstanding thermal stability (Figure 2c, red) with an initial decomposing temperature of $400{ }^{\circ} \mathrm{C}$. PPhen- $\mathrm{SO}_{3} \mathrm{H}-100 \%$ undergoes rapid weight loss (11\%) below $100{ }^{\circ} \mathrm{C}$ due to the evaporation of hydrogen-bonded $\mathrm{H}_{2} \mathrm{O}$ at $-\mathrm{SO}_{3} \mathrm{H}$ sites. At $200{ }^{\circ} \mathrm{C},-\mathrm{SO}_{3} \mathrm{H}$ starts to decompose and reaches the highest rate of weight loss at around $400{ }^{\circ} \mathrm{C}$, as indicated by the peak in the first derivatives of TGA (Figures $2 \mathrm{~d}$ and S7, Supporting Information). The weight loss in this region (16\%) is wellmatched with the $1.81 \mathrm{mmol} \cdot \mathrm{g}^{-1}$ acidic site density (corresponding to $14.5 \mathrm{wt} \%-\mathrm{SO}_{3} \mathrm{H}$ ) measured by chemical titration (Table S2, Supporting Information). Compared with the commercial catalyst Amberlyst 15 , which lost all $-\mathrm{SO}_{3} \mathrm{H}$ sites below $350{ }^{\circ} \mathrm{C},{ }^{12,39}$ the superior thermal stability of PPhen- $\mathrm{SO}_{3} \mathrm{H}-100 \%$ enables reactions catalyzed at higher temperatures. The decomposition of $-\mathrm{SO}_{3} \mathrm{H}$ in PPhen$\mathrm{SO}_{3} \mathrm{H}-0 \%$ starts at $280{ }^{\circ} \mathrm{C}$, which is $80{ }^{\circ} \mathrm{C}$ higher than that of $\mathrm{PPhen}_{-} \mathrm{SO}_{3} \mathrm{H}-100 \%$ (Figure $2 \mathrm{~d}$ ). The results agree with the literature that the stability of $-\mathrm{SO}_{3} \mathrm{H}$ increases with the decrease in crosslink content. ${ }^{12}$ There is no obvious water

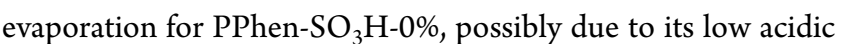
site density $\left(0.48 \mathrm{mmol} \cdot \mathrm{g}^{-1}\right)$ and porosity $\left(76 \mathrm{~m}^{2} \cdot \mathrm{g}^{-1}\right)$.

The structural changes from fresh PPhen to PPhen- $\mathrm{SO}_{3} \mathrm{H}$ are characterized by attenuated total reflection infrared (ATRIR) spectroscopy for fully crosslink polymer (Figure 3) and

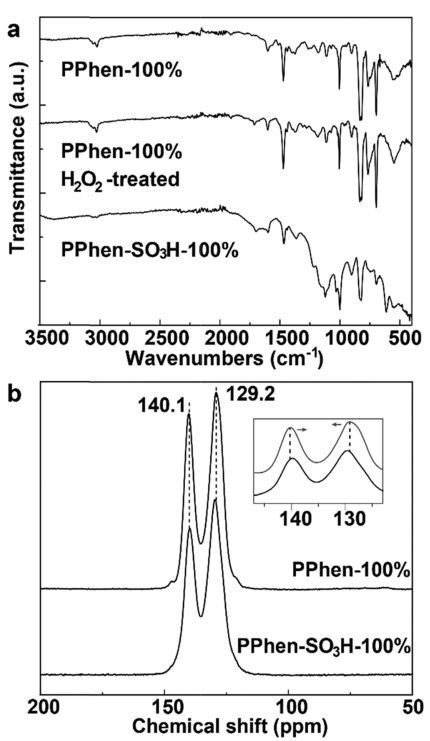

Figure 3. (a) ATR-IR spectra of fresh PPhen-100\%, $\mathrm{H}_{2} \mathrm{O}_{2}$ treated PPhen-100\%, and PPhen- $\mathrm{SO}_{3} \mathrm{H}-100 \%$. (b) Solid-state ${ }^{13} \mathrm{C}$ NMR spectra of PPhen-100\% and $\mathrm{PPhen}_{-} \mathrm{SO}_{3} \mathrm{H}-100 \%$. The inset shows the shift of NMR peaks after sulfonation.

linear polymer (Figure S8, Supporting Information). The absorption bands at around $3030 \mathrm{~cm}^{-1}$ in all spectra represent the aromatic $\mathrm{C}-\mathrm{H}$ stretch. The successful loading of $-\mathrm{SO}_{3} \mathrm{H}$ sites is confirmed by the new peaks at 1032 and $1100 \mathrm{~cm}^{-1}$, corresponding to the $\mathrm{S}=\mathrm{O}$ stretching. ${ }^{26,45,46}$ The emergence of $\mathrm{O}-\mathrm{H}$ stretching $\left(3400 \mathrm{~cm}^{-1}\right)$ and $\mathrm{O}-\mathrm{H}$ bending $(610$, $1650 \mathrm{~cm}^{-1}$ ) bands for PPhen- $\mathrm{SO}_{3} \mathrm{H}-100 \%$ shows the absorbance of $\mathrm{H}_{2} \mathrm{O}$ molecules via hydrogen bonds. ${ }^{45,46}$ This IR feature of $\mathrm{H}_{2} \mathrm{O}$ is consistent with the $\mathrm{H}_{2} \mathrm{O}$ loss in the TGA measurement, which shows $\mathrm{H}_{2} \mathrm{O}$ loss of around 3.3 per 
$-\mathrm{SO}_{3} \mathrm{H}$ ligand. All of the vibrational modes are also visible in the diffuse reflectance infrared Fourier transform spectroscopy (DRIFT) (Figure S9). The solid-state ${ }^{13} \mathrm{C}$ NMR spectrum of PPhen shows two signals at 140.1 and $129.2 \mathrm{ppm}$ in the characteristic region of $\mathrm{sp}^{2}$ carbon atoms, ${ }^{47}$ which correspond to the connecting (140.1 ppm) and nonconnecting (129.2 $\mathrm{ppm}$ ) carbons in the substituted benzene groups (Figure $3 \mathrm{~b}$ ). Although not well distinguished due to large band width, both peaks shift toward the middle after sulfonation by 0.4 to 139.7 and $129.6 \mathrm{ppm}$, respectively, suggesting the emergence of an $\mathrm{NMR}$ signal for aromatic carbon coordinated with $-\mathrm{SO}_{3} \mathrm{H}$ ligands.

Catalytic Performance of PPhen- $\mathrm{SO}_{3} \mathrm{H}$ in Phenylacetylene Hydration. We first studied the structurereactivity relationship through the hydration of aromatic alkyne phenylacetylene. The conversion of phenylacetylene and the yield of acetophenone are 22 and $16 \%$, respectively, for PPhen- $\mathrm{SO}_{3} \mathrm{H}-100 \%$ at $100{ }^{\circ} \mathrm{C}$ over $2 \mathrm{~h}$, which is 20 times higher than that of the macroreticular resin Amberlyst 15 (2030 mesh) (Figure $4 \mathrm{a}$ and Table S3). Both conversion and yield
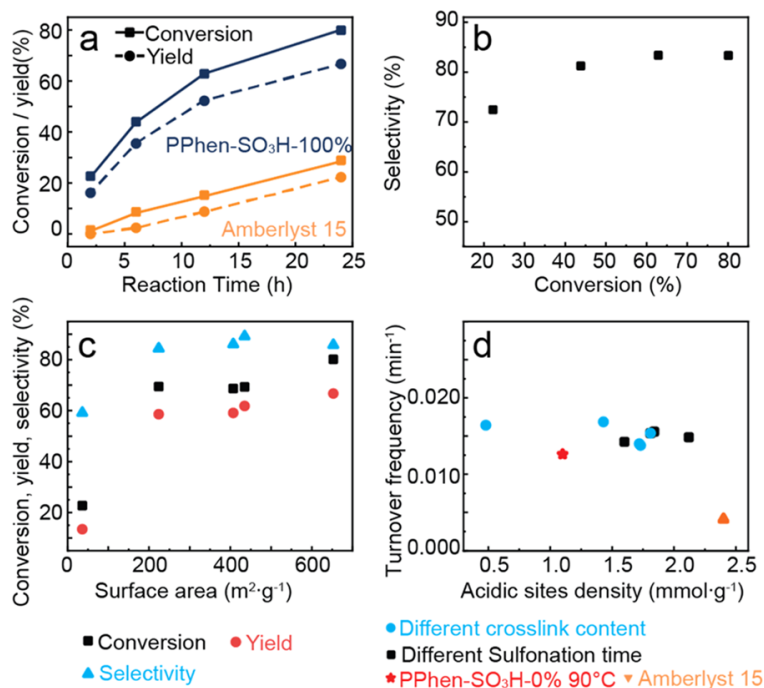

Figure 4. (a) Conversion of phenylacetylene and the yield of acetophenone as a function of time for PPhen-SO3H-100\% and Amberlyst 15. Reaction conditions: $\mathrm{H}_{2} \mathrm{O}, 1 \mathrm{~mL}$; phenylacetylene, 110 $\mu \mathrm{L}$; dodecane as the standard, $40 \mu \mathrm{L}$; catalyst, $20 \mathrm{mg}$; and temperature, $100{ }^{\circ} \mathrm{C}$. (b) Selectivity of acetophenone as the function of conversion for PPhen-SO3H-100\% at $100{ }^{\circ} \mathrm{C}$. (c) Phenylacetylene conversion, acetophenone yield, and selectivity as a function of surface area. (d) TOF as a function of acidic site density for all PPhen-based polymers (blue, black, and red) and Amberlyst 15 (orange).

gradually increase, reaching 80 and $67 \%$ at $24 \mathrm{~h}$, respectively. Overall, over $80 \%$ of selectivity is achieved between 40 and $80 \%$ conversion for PPhen- $\mathrm{SO}_{3} \mathrm{H}-100 \%$, showing superior hydration performance (Figure 4b). Ninety-five percent conversion and selectivity is reached when increasing the temperature to $120{ }^{\circ} \mathrm{C}$. The catalytic activities of the commercial gel-type resins are also evaluated under the same reaction conditions. The conversions of phenylacetylene are $5.2,15$, and $20 \%$ for three chosen resins (Dowex 50WX2, Dowex G26, Dowex Marathon), respectively (Table S4, Supporting Information), which again shows the potential of PPhen- $\mathrm{SO}_{3} \mathrm{H}$ as a promising solid acid.
PPhen- $\mathrm{SO}_{3} \mathrm{H}$ catalysts show an increase in phenylacetylene conversion from 23 to $80 \%$ when the surface area increases from 36 to $653 \mathrm{~m}^{2} \cdot \mathrm{g}^{-1}$, corresponding to an increase in crosslink content from 0 to $100 \%$ (Figure 4c). Similarly, the yield of acetophenone increases from 13 to $67 \%$. The positive relationship between activity and crosslink content is different from conventional polymeric solid acid systems, in which catalysts with less crosslink content usually have higher activity due to the flexibility of the polymer frameworks, which allows the diffusion of reactants and products. ${ }^{12,31,48}$ Studies on polystyrene-divinylbenzene-based solid acids showed that the diffusion coefficient through the polymer framework decreased strongly above $8 \%$ divinylbenzene content. ${ }^{31}$ In comparison, PPhen- $\mathrm{SO}_{3} \mathrm{H}-100 \%$ is stacked via $\pi-\pi$ interactions, which can swell at the $z$ axis, offering high accessibility of phenylacetylene, even at high crosslink content (Scheme 1). It achieves $1.81 \mathrm{mmol} \cdot \mathrm{g}^{-1}$ of acid density with sulfuric acid at 40 ${ }^{\circ} \mathrm{C}$, which is much milder than conventional sulfonation conditions, including the use of oleum or $\mathrm{SO}_{3}$ at elevated temperatures. $^{12,48,49}$

To justify the increasing catalytic activity with higher crosslink content, $\mathrm{PPhen}-\mathrm{SO}_{3} \mathrm{H}$ catalysts with a range of acidic site density are investigated. They include the aforementioned PPhen- $\mathrm{SO}_{3} \mathrm{H}$ catalysts with varied crosslink contents (Figure $4 \mathrm{~d}$, blue circle), PPhen- $\mathrm{SO}_{3} \mathrm{H}-100 \%$ with different sulfonation durations (Figure $4 \mathrm{~d}$, black square), and $\mathrm{PPhen}-\mathrm{SO}_{3} \mathrm{H}-0 \%$ with higher sulfonation temperatures (Figure $4 \mathrm{~d}$, red star). Amberlyst 15 is also included as a comparison (Figure 4d, orange triangle). Determined by titration, the acidic site density is $0.48 \mathrm{mmol} \cdot \mathrm{g}^{-1}$ for $\mathrm{PPhen}-\mathrm{SO}_{3} \mathrm{H}-0 \%$, which increases to $1.43,1.73,1.72$, and $1.81 \mathrm{mmol} \cdot \mathrm{g}^{-1}$ for PPhen $-\mathrm{SO}_{3} \mathrm{H}-36 \%$, PPhen- $\mathrm{SO}_{3} \mathrm{H}-65 \%$, PPhen- $\mathrm{SO}_{3} \mathrm{H}-82 \%$, and PPhen- $\mathrm{SO}_{3} \mathrm{H}-$ $100 \%$, respectively (Table S2, Supporting Information). This shows that higher porosity enables higher $-\mathrm{SO}_{3} \mathrm{H}$ site density, as $-\mathrm{SO}_{3} \mathrm{H}$ groups are more easily grafted onto the polymer frameworks. ${ }^{50}$ With extended sulfonation duration, the highest density of $2.12 \mathrm{mmol} \cdot \mathrm{g}^{-1}$ is achieved (Table S2, Supporting Information), corresponding to one $-\mathrm{SO}_{3} \mathrm{H}$ site per 4.5 substituted benzene groups. Under the same reaction condition, similar TOF at $0.015 \pm 0.001 \mathrm{~min}^{-1}$ is achieved for all PPhen- $\mathrm{SO}_{3} \mathrm{H}$ catalysts at different acidic densities and crosslink contents (Figure 4d), suggesting that PPhen- $\mathrm{SO}_{3} \mathrm{H}$ offers high accessibility to both $\mathrm{H}_{2} \mathrm{O}$ and phenylacetylene molecules during the reaction. This is attributed to the high swellability of PPhen- $\mathrm{SO}_{3} \mathrm{H}$ even at $100 \%$ crosslink content. In comparison, the TOF for Amberlyst 15 is only $0.004 \mathrm{~min}^{-1}$ (Figure 4d).

The $-\mathrm{SO}_{3} \mathrm{H}$ ligands also exhibit stability on the series of PPhen- $\mathrm{SO}_{3} \mathrm{H}$ solid acids, in which PPhen- $\mathrm{SO}_{3} \mathrm{H}-100 \%$ maintains phenylacetylene conversion in the range of 60$75 \%$ for three cycles with over $85 \%$ selectivity toward acetophenone (Figure S10). The generally lower selectivity for all tested catalysts is due to the carbon balance problem associated with the reaction system.

Alkyne Activation over PPhen. PPhen- $\mathrm{SO}_{3} \mathrm{H}$ catalysts also benefit from a high density of phenylene and tetrasubstituted benzene groups, for which the interaction with alkynes is studied via infrared spectra (Figure 5) by absorbing a small number of reactants within the PPhen- $\mathrm{SO}_{3} \mathrm{H}$ catalysts (Figure 5a). The peaks for $\mathrm{C} \equiv \mathrm{C}$ bond (2100-2260 $\mathrm{cm}^{-1}$ ) are difficult to identify due to weak intensity, while alkyne $\mathrm{C}-\mathrm{H}$ stretching is identified at around $3289 \mathrm{~cm}^{-1}$ for pure phenylacetylene. It is red-shifted to $3281 \mathrm{~cm}^{-1}$ for 

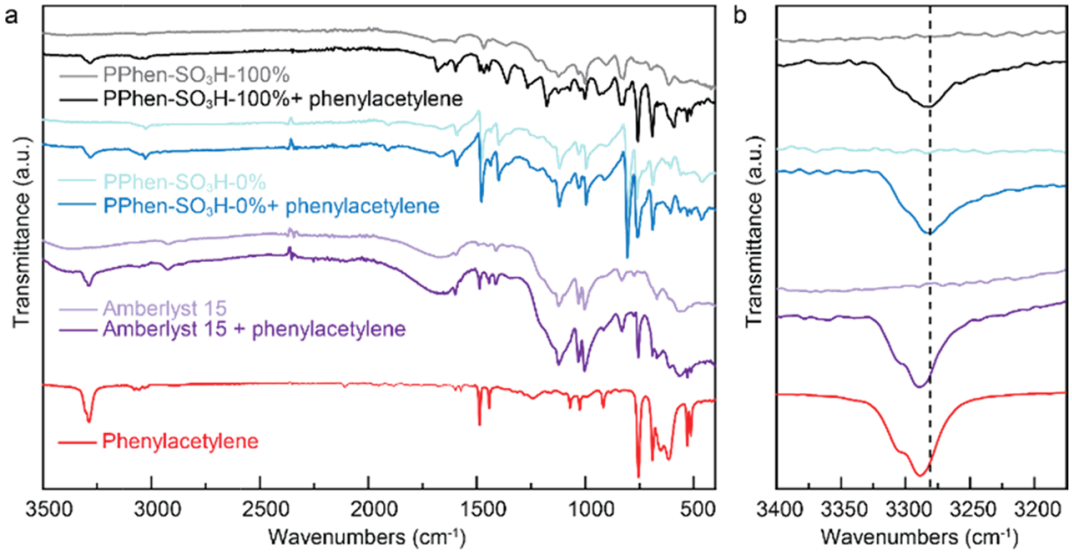

Figure 5. (a) ATR-IR spectra for PPhen- $\mathrm{SO}_{3} \mathrm{H}-100 \%$, $\mathrm{PPhen}-\mathrm{SO}_{3} \mathrm{H}-0 \%$, and Amberlyst 15 catalysts showing their interaction with phenylacetylene. (b) Zoom-in of (a) with the wavenumber between 3400 and $3175 \mathrm{~cm}^{-1}$.

phenylacetylene over both PPhen- $\mathrm{SO}_{3} \mathrm{H}-100 \%$ and PPhen$\mathrm{SO}_{3} \mathrm{H}-0 \%$. In comparison, the alkyne $\mathrm{C}-\mathrm{H}$ stretching of phenylacetylene over Amberlyst 15 remains at $3289 \mathrm{~cm}^{-1}$. This effect can be understood as the interaction between the substituted benzene groups in the PPhen and the aromatic alkyne in Amberlyst 15 with an edge-to-face (T-shape) configuration (Scheme 1, right), in which phenylacetylene is vertically adsorbed on the alkynyl group $(-\mathrm{C} \equiv \mathrm{C}-\mathrm{H})$ pointing to the substituted benzene group to minimize the repulsive force with the adjacent framework benzene groups. As a result, the $\mathrm{C} \equiv \mathrm{C}$ bond is weakened and shifts the alkyne $\mathrm{C}-\mathrm{H}$ peak toward a lower frequency region. The existence of this configuration is rationalized by research on the $\mathrm{CH} / \pi$ interaction between benzene and hydrocarbons, which showed the preference of $\mathrm{C}-\mathrm{H}$ bonds for pointing toward the benzene ring and that the benzene-acetylene complex bore the strongest interaction among all complexes studied. ${ }^{35}$ The high density of phenylene and tetrasubstituted benzene groups in $\mathrm{PPhen}-\mathrm{SO}_{3} \mathrm{H}$ catalysts maximizes such absorption and activation of phenylacetylene.

In addition, $\mathrm{PPhen}-\mathrm{SO}_{3} \mathrm{H}$ can be well dispersed in water, in which the hydrophilicity of the $-\mathrm{SO}_{3} \mathrm{H}$ group and hydrophobicity of the PPhen framework (Figure S11 and Movies S1 and S2, Supporting Information) enable the simultaneous adsorption of $\mathrm{H}_{2} \mathrm{O}$ and alkynes. This creates a "solvent-like" environment, which enables alkynes to accumulate around the surface of PPhen and facilitates the subsequent reaction with hydrogen-bonded water at $-\mathrm{SO}_{3} \mathrm{H}$ sites. The excess amount of water will further dilute the local concentration of the product ketone as it is more soluble than alkynes and drive the reaction forward.

Hydration of Aliphatic Alkynes. The $\mathrm{CH} / \pi$ interaction is not limited to aromatic alkynes, as it is proposed by studies on direct interaction between aliphatic substituents and the counterpart aromatic groups. ${ }^{51-56}$ Therefore, we propose that PPhen- $\mathrm{SO}_{3} \mathrm{H}$ can also activate aliphatic alkynes. The hydration of 1-octyne was studied with PPhen- $\mathrm{SO}_{3} \mathrm{H}-100 \%$ catalysts with the highest acidic site density $\left(2.12 \mathrm{mmol} \cdot \mathrm{g}^{-1}\right)$ and Amberlyst $15\left(2.4 \mathrm{mmol} \cdot \mathrm{g}^{-1}\right)$. PPhen- $\mathrm{SO}_{3} \mathrm{H}-100 \%$ obtains a conversion of $51 \%$ and a yield of $30 \%$ as expected, while Amberlyst 15 shows almost no activity toward the hydration of the aliphatic alkyne (Figure 6a), again showing the exceptional activation of alkynes over a PPhen framework. The reduced activity compared with the phenylacetylene substrate can be understood as the lack of the conjugation between phenyl and $\mathrm{C} \equiv \mathrm{C}$

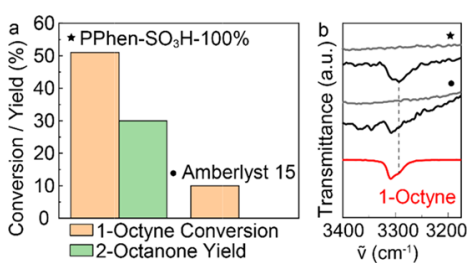

Figure 6. (a) Conversion of 1-octyne and the yield of 2-octanone. Reaction conditions: $0.2 \mathrm{mmol}$ of acidic sites, $100 \mathrm{mg}$ of PPhen$\mathrm{SO}_{3} \mathrm{H}-100 \%$ or $83 \mathrm{mg}$ of Amberlyst, $0.5 \mathrm{mmol}$ of 1 -octyne, $1 \mathrm{~mL}$ of $\mathrm{H}_{2} \mathrm{O}, 24 \mathrm{~h}$, and $120^{\circ} \mathrm{C}$. (b) ATR-IR spectra between wavenumber 3400 and $3175 \mathrm{~cm}^{-1}$ for PPhen- $\mathrm{SO}_{3} \mathrm{H}-100 \%$ and Amberlyst 15 catalysts showing their interaction with 1-octyne.

groups, which activate the $\mathrm{C} \equiv \mathrm{C}$ in phenylacetylene. The interaction between the PPhen framework and 1-octyne is also studied with ATR-IR (Figures 6b and S12, Supporting Information), which shows a red shift of the $\mathrm{C}-\mathrm{H}$ stretching peak from $3309 \mathrm{~cm}^{-1}$ for pure 1-octyne and that adsorbed in Amberlyst 15 to $3293 \mathrm{~cm}^{-1}$.

\section{CONCLUSIONS}

$\mathrm{SO}_{3} \mathrm{H}$-group functionalized PPhen is designed with adjustable crosslink contents and acidic site densities. The advancement of such catalysts features high porosity, which allows environment-friendly synthesis using a mild sulfonation process, as well as excellent swellability, which ensures accessibility of all acidic sites in the pores. The phenylene and tetrasubstituted benzene groups can activate reactants through $\mathrm{CH} / \pi$ interactions with a T-shape configuration and provide "an organic solvent-like environment". Being able to activate aliphatic $\mathrm{C} \equiv \mathrm{C}$ under mild conditions, PPhen- $\mathrm{SO}_{3} \mathrm{H}$ serves as a promising candidate for alkyne chemistry in the replacement of highly toxic liquid acids in the synthesis of valuable chemicals.

\section{ASSOCIATED CONTENT}

\section{Supporting Information}

The Supporting Information is available free of charge at https://pubs.acs.org/doi/10.1021/acs.chemmater.0c01763.

Calculation of TOF, acidic site density, and crosslink content; characterizations of PPhen- $\mathrm{SO}_{3} \mathrm{H}-0 \%$ and PPhen- $\mathrm{SO}_{3} \mathrm{H}-100 \%$; catalytic performance of PPhen$\mathrm{SO}_{3} \mathrm{H}$ and commercial acidic resins (PDF) 
Hydrophobicity of PPhen, Movie S1_S-PPhen (MP4) Hydrophobicity of PPhen- $\mathrm{SO}_{3} \mathrm{H}$, Movie S2_PPhen (MP4)

\section{AUTHOR INFORMATION}

\section{Corresponding Author}

Feng Ryan Wang - Department of Chemical Engineering, University College London, London WC1E 7JE, U.K.; ๑ orcid.org/0000-0002-2475-606X; Email: ryan.wang@ ucl.ac.uk

\section{Authors}

Yiyun Liu - Department of Chemical Engineering, University College London, London WC1E 7JE, U.K.; $\odot$ orcid.org/00000003-1853-6105

Bolun Wang - Department of Chemical Engineering, University College London, London WC1E 7JE, U.K.

Liqun Kang - Department of Chemical Engineering, University College London, London WC1E 7JE, U.K.; $\odot$ orcid.org/00000003-2100-4310

Apostolos Stamatopoulos - Department of Chemical Engineering, University College London, London WC1E 7JE, U.K.

Hao Gu - Department of Chemical Engineering, University College London, London WC1E 7JE, U.K.

Complete contact information is available at: https://pubs.acs.org/10.1021/acs.chemmater.0c01763

\section{Notes}

The authors declare no competing financial interest.

\section{ACKNOWLEDGMENTS}

This project is funded by EPSRC (EP/P02467X/1 and EP/ S018204/2), Royal Society (RG160661, IES $\backslash R 3 \backslash 170097$, IES $\backslash R 1 \backslash 191035)$, and the Newton Fellowship (NF170761). We acknowledge Diamond Light Source beamtime (EM22572 and EM21641). This research has been performed with the use of facilities at the Research Complex at Harwell, including DRIFTS equipment. The authors would like to thank the Research Complex for access and support to these facilities and equipment.

\section{REFERENCES}

(1) Wood, C. D.; Tan, B.; Trewin, A.; Su, F.; Rosseinsky, M. J.; Bradshaw, D.; Sun, Y.; Zhou, L.; Cooper, A. I. Microporous organic polymers for methane storage. Adv. Mater. 2008, 20, 1916-1921.

(2) Du, N.; Robertson, G. P.; Song, J.; Pinnau, I.; Thomas, S.; Guiver, M. D. Polymers of intrinsic microporosity containing trifluoromethyl and phenylsulfone groups as materials for membrane gas separation. Macromolecules 2008, 41, 9656-9662.

(3) Abidian, M. R.; Kim, D. H.; Martin, D. C. Conducting-polymer nanotubes for controlled drug release. Adv. Mater. 2006, 18, 405-409.

(4) Zhao, C.; Danish, E.; Cameron, N. R.; Kataky, R. Emulsiontemplated porous materials (PolyHIPEs) for selective ion and molecular recognition and transport: applications in electrochemical sensing. J. Mater. Chem. 2007, 17, 2446-2453.

(5) Schacher, F.; Ulbricht, M.; Müller, A. H. Self-supporting, double stimuli-responsive porous membranes from polystyrene-block-poly (N, N-dimethylaminoethyl methacrylate) diblock copolymers. Adv. Funct. Mater. 2009, 19, 1040-1045.

(6) Wu, D.; Xu, F.; Sun, B.; Fu, R.; He, H.; Matyjaszewski, K. Design and Preparation of Porous Polymers. Chem. Rev. 2012, 112, 39594015.
(7) Sun, Q.; Dai, Z.; Meng, X.; Xiao, F.-S. Porous polymer catalysts with hierarchical structures. Chem. Soc. Rev. 2015, 44, 6018-6034.

(8) Sakaushi, K.; Nickerl, G.; Wisser, F. M.; Nishio-Hamane, D.; Hosono, E.; Zhou, H.; Kaskel, S.; Eckert, J. An energy storage principle using bipolar porous polymeric frameworks. Angew. Chem., Int. Ed. 2012, 51, 7850-7854.

(9) Kuhn, P.; Antonietti, M.; Thomas, A. Porous, covalent triazinebased frameworks prepared by ionothermal synthesis. Angew. Chem., Int. Ed. 2008, 47, 3450-3453.

(10) Palkovits, R.; Antonietti, M.; Kuhn, P.; Thomas, A.; Schuth, F. Solid Catalysts for the Selective Low-Temperature Oxidation of Methane to Methanol. Angew. Chem., Int. Ed. 2009, 48, 6909-6912.

(11) Sidorov, S. N.; Volkov, I. V.; Davankov, V. A.; Tsyurupa, M. P.; Valetsky, P. M.; Bronstein, L. M.; Karlinsey, R.; Zwanziger, J. W.; Matveeva, V. G.; Sulman, E. M.; Lakina, N. V.; Wilder, E. A.; Spontak, R. J. Platinum-Containing Hyper-Cross-Linked Polystyrene as a Modifier-Free Selective Catalyst for l-Sorbose Oxidation. J. Am. Chem. Soc. 2001, 123, 10502-10510.

(12) Richter, F. H.; Pupovac, K.; Palkovits, R.; Schüth, F. Set of Acidic Resin Catalysts To Correlate Structure and Reactivity in Fructose Conversion to 5-Hydroxymethylfurfural. ACS Catal. 2013, 3, 123-127.

(13) Zhang, Y.; Wei, S.; Liu, F.; Du, Y.; Liu, S.; Ji, Y.; Yokoi, T.; Tatsumi, T.; Xiao, F.-S. Superhydrophobic nanoporous polymers as efficient adsorbents for organic compounds. Nano Today 2009, 4, 135-142.

(14) Wang, G.-H.; Sun, Q.; Zhang, R.; Li, W.-C.; Zhang, X.-Q.; Lu, A.-H. Weak Acid-Base Interaction Induced Assembly for the Synthesis of Diverse Hollow Nanospheres. Chem. Mater. 2011, 23, 4537-4542.

(15) Chen, L.; Yang, Y.; Jiang, D. CMPs as Scaffolds for Constructing Porous Catalytic Frameworks: A Built-in Heterogeneous Catalyst with High Activity and Selectivity Based on Nanoporous Metalloporphyrin Polymers. J. Am. Chem. Soc. 2010, 132, 9138-9143.

(16) Mackintosh, H. J.; Budd, P. M.; McKeown, N. B. Catalysis by microporous phthalocyanine and porphyrin network polymers. J. Mater. Chem. 2008, 18, 573-578.

(17) Liu, F.; Wang, L.; Sun, Q.; Zhu, L.; Meng, X.; Xiao, F.-S. Transesterification Catalyzed by Ionic Liquids on Superhydrophobic Mesoporous Polymers: Heterogeneous Catalysts That Are Faster than Homogeneous Catalysts. J. Am. Chem. Soc. 2012, 134, 16948-16950.

(18) Rinaldi, R.; Palkovits, R.; Schüth, F. Depolymerization of Cellulose Using Solid Catalysts in Ionic Liquids. Angew. Chem., Int. Ed. 2008, 47, 8047-8050.

(19) Izumi, Y. Hydration/hydrolysis by solid acids. Catal. Today 1997, 33, 371-409.

(20) Liang, S.; Hammond, G. B.; Xu, B. Efficient hydration of alkynes through acid-assisted Bronsted acid catalysis. Chem. Commun. 2015, 51, 903-906.

(21) Tanemura, K.; Suzuki, T. Hydration of aromatic terminal alkynes catalyzed by sulfonated condensed polynuclear aromatic (SCOPNA) resin in water. Tetrahedron Lett. 2017, 58, 955-958.

(22) Chai, S.-H.; Wang, H.-P.; Liang, Y.; Xu, B.-Q. Sustainable production of acrolein: gas-phase dehydration of glycerol over 12tungstophosphoric acid supported on $\mathrm{ZrO} 2$ and $\mathrm{SiO} 2$. Green Chem. 2008, 10, 1087-1093.

(23) Chai, S. H.; Wang, H. P.; Liang, Y.; Xu, B. Q. Sustainable production of acrolein: investigation of solid acid-base catalysts for gas-phase dehydration of glycerol. Green Chem. 2007, 9, 1130-1136.

(24) Tang, H.; Li, N.; Li, G. Y.; Wang, W. T.; Wang, A. Q.; Cong, Y.; Wang, X. D. Dehydration of Carbohydrates to 5-Hydroxymethylfurfural over Lignosulfonate-Based Acidic Resin. ACS Sustainable Chem. Eng. 2018, 6, 5645-5652.

(25) Liu, F.; Sun, J.; Zhu, L.; Meng, X.; Qi, C.; Xiao, F.-S. Sulfated graphene as an efficient solid catalyst for acid-catalyzed liquid reactions. J. Mater. Chem. 2012, 22, 5495-5502.

(26) Xing, R.; Liu, N.; Liu, Y. M.; Wu, H. W.; Jiang, Y. W.; Chen, L.; He, M. Y.; Wu, P. Novel solid acid catalysts: Sulfonic acid group- 
functionalized mesostructured polymers. Adv. Funct. Mater. 2007, 17, $2455-2461$.

(27) Alonso, F.; Beletskaya, I. P.; Yus, M. Transition-MetalCatalyzed Addition of Heteroatom-Hydrogen Bonds to Alkynes. Chem. Rev. 2004, 104, 3079-3160.

(28) Anastas, P. T.; Williamson, T. C. Green Chemistry: Frontiers in Benign Chemical Syntheses and Processes; Oxford University Press: USA, 1998.

(29) Kutscheroff, M. Ueber eine neue Methode direkter Addition von Wasser (Hydratation) an die Kohlenwasserstoffe der Acetylenreihe. Ber. Dtsch. Chem. Ges. 1881, 14, 1540-1542.

(30) Pietrzyk, D. J.; Marcus, Y. Ion Exchanger-Solvent Interactions: Properties and Methodology. CRC Crit. Rev. Anal. Chem. 1976, 6, $131-170$.

(31) Rodriguez, O.; Setínek, K. Dependence of esterification rates on crosslinking of ion exchange resins used as solid catalysts. J. Catal. 1975, 39, 449-455.

(32) He, L.; Cornelius, C. J.; Perahia, D. Water dynamics within a highly rigid sulfonated polyphenylene. Eur. Polym. J. 2014, 56, 168173.

(33) He, L.; Fujimoto, C. H.; Cornelius, C. J.; Perahia, D. From Solutions to Membranes: Structure Studies of Sulfonated Polyphenylene Ionomers. Macromolecules 2009, 42, 7084-7090.

(34) Wang, F.; Mielby, J.; Richter, F. H.; Wang, G.; Prieto, G.; Kasama, T.; Weidenthaler, C.; Bongard, H. J.; Kegnaes, S.; Furstner, A.; Schuth, F. A polyphenylene support for Pd catalysts with exceptional catalytic activity. Angew. Chem., Int. Ed. 2014, 53, 86458648.

(35) Tsuzuki, S.; Honda, K.; Uchimaru, T.; Mikami, M.; Tanabe, K. The Magnitude of the $\mathrm{CH} / \pi$ Interaction between Benzene and Some Model Hydrocarbons. J. Am. Chem. Soc. 2000, 122, 3746-3753.

(36) Dreyer, D. R.; Jia, H. P.; Bielawski, C. W. Graphene oxide: a convenient carbocatalyst for facilitating oxidation and hydration reactions. Angew. Chem., Int. Ed. 2010, 122, 6965-6816.

(37) Fischer, J.; Hölderich, W. F. Baeyer-Villiger-oxidation of cyclopentanone with aqueous hydrogen peroxide by acid heterogeneous catalysis. Appl. Catal., A 1999, 180, 435-443.

(38) Boz, N.; Degirmenbasi, N.; Kalyon, D. M. Esterification and transesterification of waste cooking oil over Amberlyst 15 and modified Amberlyst 15 catalysts. Appl. Catal., B 2015, 165, 723-730.

(39) Fan, G.; Liao, C.; Fang, T.; Luo, S.; Song, G. Amberlyst 15 as a new and reusable catalyst for the conversion of cellulose into cellulose acetate. Carbohydr. Polym. 2014, 112, 203-209.

(40) Sakurai, H.; Koga, K.; Kiuchi, M. Gold nanoparticles deposited on Amberlyst-15: Metal-acid bifunctional catalyst for cellobiose conversion to gluconic acid. Catal. Today 2015, 251, 96-102.

(41) Rose, M.; Klein, N.; Senkovska, I.; Schrage, C.; Wollmann, P.; Böhlmann, W.; Böhringer, B.; Fichtner, S.; Kaskel, S. A new route to porous monolithic organic frameworks via cyclotrimerization. J. Mater. Chem. 2011, 21, 711-716.

(42) Meng, Y.; Gu, D.; Zhang, F. Q.; Shi, Y. F.; Cheng, L.; Feng, D.; Wu, Z. X.; Chen, Z. X.; Wan, Y.; Stein, A.; Zhao, D. Y. A family of highly ordered mesoporous polymer resin and carbon structures from organic-organic self-assembly. Chem. Mater. 2006, 18, 4447-4464.

(43) Zhang, F. Q.; Meng, Y.; Gu, D.; Yan, Y.; Yu, C. Z.; Tu, B.; Zhao, D. Y. A facile aqueous route to synthesize highly ordered mesoporous polymers and carbon frameworks with Ia(3)over-bard bicontinuous cubic structure. J. Am. Chem. Soc. 2005, 127, 1350813509 .

(44) Meng, Y.; Gu, D.; Zhang, F. Q.; Shi, Y. F.; Yang, H. F.; Li, Z.; Yu, C. Z.; Tu, B.; Zhao, D. Y. Ordered mesoporous polymers and homologous carbon frameworks: Amphiphilic surfactant templating and direct transformation. Angew. Chem., Int. Ed. 2005, 44, 70537059.

(45) Yadav, G. D.; Murkute, A. D. Preparation of the Novel Mesoporous Solid Acid Catalyst UDCaT-4 via Synergism of Persulfated Alumina and Zirconia into Hexagonal Mesoporous Silica for Alkylation Reactions. Adv. Synth. Catal. 2004, 346, 389-394.
(46) Zhou, W.; Yoshino, M.; Kita, H.; Okamoto, K.-i. Carbon Molecular Sieve Membranes Derived from Phenolic Resin with a Pendant Sulfonic Acid Group. Ind. Eng. Chem. Res. 2001, 40, 48014807.

(47) Baccile, N.; Laurent, G.; Babonneau, F.; Fayon, F.; Titirici, M.M.; Antonietti, M. Structural Characterization of Hydrothermal Carbon Spheres by Advanced Solid-State MAS 13C NMR Investigations. J. Phys. Chem. C 2009, 113, 9644-9654.

(48) Casas, C.; Bringue, R.; Ramirez, E.; Iborra, M.; Tejero, J. Liquid-phase dehydration of 1-octanol, 1-hexanol and 1-pentanol to linear symmetrical ethers over ion exchange resins. Appl. Catal., A 2011, 396, 129-139.

(49) Agirrezabal-Telleria, I.; Requies, J.; Güemez, M. B.; Arias, P. L. Pore size tuning of functionalized SBA-15 catalysts for the selective production of furfural from xylose. Appl. Catal., B 2012, 115-116, $169-178$

(50) Struck, M.; Widdecke, H. Surface functionalization of polymer networks. Sulfonation of the internal surface of macroporous styrenedivinylbenzene copolymers. Angew. Makromol. Chem. 1996, 235, $131-148$.

(51) Wheeler, S. E.; Houk, K. N. Substituent Effects in Cation $/ \pi$ Interactions and Electrostatic Potentials above the Centers of Substituted Benzenes Are Due Primarily to Through-Space Effects of the Substituents. J. Am. Chem. Soc. 2009, 131, 3126-3127.

(52) Wheeler, S. E.; Houk, K. N. Substituent Effects in the Benzene Dimer are Due to Direct Interactions of the Substituents with the Unsubstituted Benzene. J. Am. Chem. Soc. 2008, 130, 10854-10855.

(53) Rashkin, M. J.; Waters, M. L. Unexpected Substituent Effects in Offset $\pi-\pi$ Stacked Interactions in Water. J. Am. Chem. Soc. 2002, $124,1860-1861$

(54) Bloom, J. W. G.; Wheeler, S. E. Taking the Aromaticity out of Aromatic Interactions. Angew. Chem. 2011, 123, 7993-7995.

(55) Grimme, S. Do special noncovalent $\pi-\pi$ stacking interactions really exist? Angew. Chem., Int. Ed. 2008, 47, 3430-3434.

(56) Paliwal, S.; Geib, S.; Wilcox, C. S. Molecular Torsion Balance for Weak Molecular Recognition Forces. Effects of "Tilted-T" Edgeto-Face Aromatic Interactions on Conformational Selection and Solid-State Structure. J. Am. Chem. Soc. 1994, 116, 4497-4498. 